\title{
Multiparameter Investigation of a $46, X X / 46, X Y$ Tetragametic Chimeric Phenotypical Male Patient with Bilateral Scrotal Ovotestes and Ovulatory Activity
}

\author{
Yolande van Bever ${ }^{a, g} \quad$ Katja P. Wolffenbuttel ${ }^{b, g} \quad$ Hennie T. Brüggenwirth $^{\text {a, }}$ \\ Eric Blom ${ }^{\mathrm{a}, \mathrm{g}}$ Annelies de Klein ${ }^{\mathrm{a}}$ h Bert H.J. Eussen ${ }^{\mathrm{a}}$ Florijn van der Windt $^{\mathrm{h}}$ \\ Sabine E. Hannemac,g Arianne B. Dessens ${ }^{d, g}$ Lambert C.J. Dorssers $^{\mathrm{e}}$ \\ Katharina Biermann ${ }^{e}$ Remko Hersmus ${ }^{e, h}$ Yolanda B. de Rijke, g \\ Leendert H.J. Looijenga ${ }^{\mathrm{e}} \mathrm{g}$ \\ Departments of a Clinical Genetics, ${ }^{b}$ Urology and Pediatric Urology, ${ }^{c}$ Pediatric Endocrinology, ${ }^{d}$ Child and Adolescent \\ Psychiatry and Psychology, ePathology, Laboratory of Experimental Patho-Oncology, and ${ }^{\mathrm{f} C l i n i c a l}$ Chemistry and \\ 9DSD Team Rotterdam, Erasmus Medical Center, Rotterdam, and h Department of Urology, Tergooi Hospitals, \\ Hilversum, The Netherlands
}

\section{Keywords}

Blood typing - DNA polymorphisms - Hormonal treatment . Mosaicism · Next-generation sequencing · Ovotestis . Ovulatory activity · (Partial) gonadectomy · SNP array . $\mathrm{XX} / \mathrm{XY}$ chimerism

\section{Abstract}

We report on an adult male initially presenting with gynecomastia and a painless scrotal mass without additional genital anomalies. Hyperpigmentation of the skin following the Blaschko's lines was identified. He underwent gonadectomy because of suspected cancer. Histological analyses revealed an ovotestis with ovulatory activity confirmed by immunohistochemistry with multiple markers. Karyotyping of cultured peripheral blood lymphocytes and a buccal smear revealed a 46,XX/46,XY chimeric constitution with different percentages. Multiple molecular analyses as well as blood typing implied a tetragametic origin. After the unilateral go-
\end{abstract}

\section{KARGER}

E-Mail karger@karger.com www.karger.com/sxd

\section{(C) 2017 The Author(s)}

Published by S. Karger AG, Basel

Karger

Open access

This article is licensed under the Creative Commons AttributionNonCommercial-NoDerivatives 4.0 International License (CC BY NC-ND) (http://www.karger.com/Services/OpenAccessLicense). Usage and distribution for commercial purposes as well as any distribution of modified material requires written permission. nadectomy, the patient developed recurrent painful cystic swellings of the remaining gonad. Because of the wish to preserve hormonal activity as well as future fertility, the patient underwent surgical resection of a cystic gonadal area. The removed tissue showed ovulation-related features in addition to both testicular and ovarian tissue, diagnosed as an ovotestis. Testosterone therapy was initiated to suppress the persistently elevated gonadotropins and thereby suppress ovarian activity. During treatment, the recurrent pain complaints and cystic swellings ceased, although gonadotropin levels were not fully suppressed. Based on these observations, the importance of a detailed genetic and pathological diagnosis and the clinical dilemmas including the pros and cons of personalized treatment with gonadal preservative surgery are discussed.

(0) 2017 The Author(s) Published by S. Karger AG, Basel

Y.V.B. and K.P.W. contributed equally to this work.
Yolande van Bever

Department of Clinical Genetics, Erasmus Medical Center 's-Gravendijkwal 230

NL-3015 CE Rotterdam (The Netherlands)

E-Mail y.vanbever@erasmusmc.nl 
The genetic basis of specific types of disorders of sex development (DSD) remains partly unexplained, which can be due to various reasons, including absence of informative data as well as the actual inability to interpret the data if present. This also applies to possible chimerism, defined as the presence of 2 separate genetic cell lines within a single individual. It can be limited to a single tissue or affect multiple or even all tissues [Lee et al., 2014; Xia et al., 2014]. A number of clinical interventions can cause chimerism, including transplantation and prenatal transfusion. Alternatively, it can be related to a disturbed fertilization and may have a dispermic, tetragametic, or parthogenetic origin. The actual prevalence of these alternative mechanisms is unknown, as it is expected that many of the affected individuals are in fact healthy and are only incidentally identified for example by blood group or paternity/maternity testing or other clinical investigations
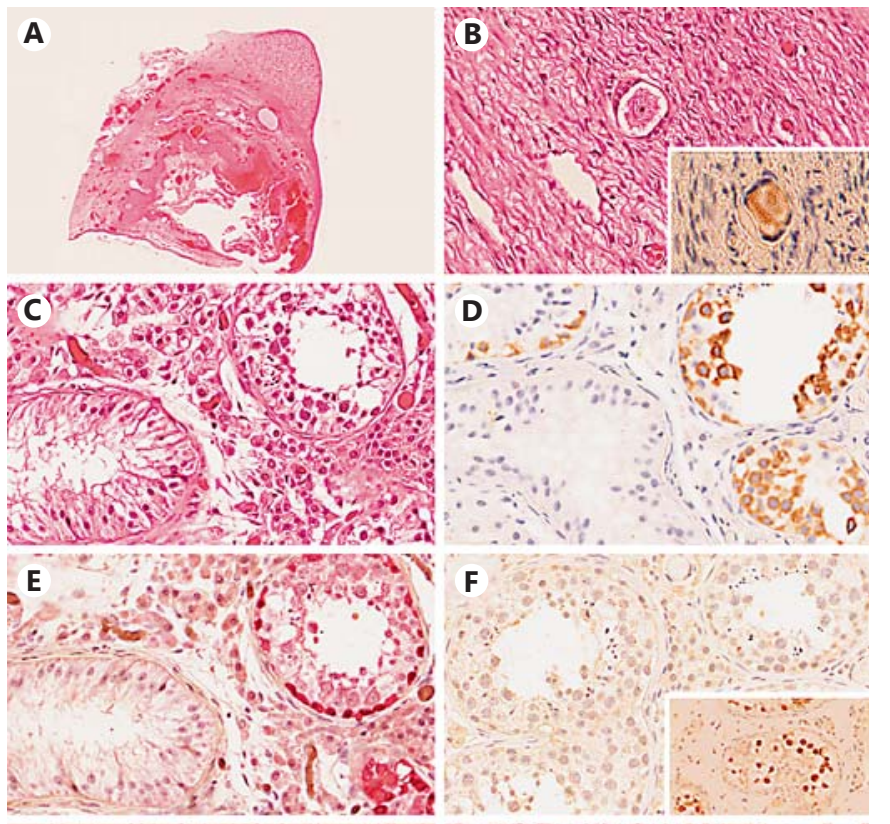

$\mathbf{F}$
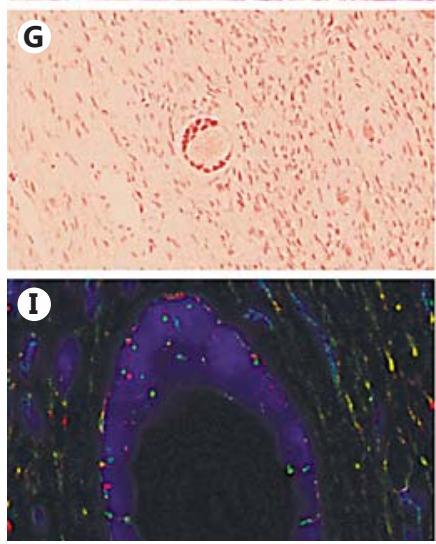

related to health issues [Zhu et al., 2014]. However, detection of chimerism has become feasible with the application of targeted as well as genome-wide single nucleotide polymorphism (SNP) analyses, as recently demonstrated [Conlin et al., 2010].

Identification of the presence of chimerism can be relevant in the context of elucidation of the genetic mechanism underlying various forms of DSD, including ovotesticular-DSD (OT-DSD). This is a rare form of DSD, diagnosed on defined histological characteristics of gonadal tissue. Specifically, both testicular and ovarian tissue must be present in a single patient, although not restricted to a single gonad. Importantly, the testicular tissue can be devoid of germ cells, while the presence of (female) germ cells (oogonia/oocytes) is a prerequisite for the classification of the ovarian component. These definitions must be followed strictly to prevent overdiagnosis of this variant of DSD with clinical implications. It is not easy to identify patients with OT-DSD based on the genital phenotype, because this may vary from ambiguous to atypical to normal genitalia. Also at the level of chromosomal and molecular constitution, patients with OT-DSD can be highly heterogeneous. About $60 \%$ have a 46,XX karyotype, which was recently suggested to be due to mutations in NR5A1 [Baetens et al., 2016; Bashamboo et al., 2016; Igarashi et al., 2017]. A cytoge-

Fig. 1. Histology and immunohistochemistry of the gonad after gonadectomy demonstrating an ovotestis. A Representative hematoxylin and eosin (HE) staining showing an overview of the whole gonad. Testicular tissue is present in the upper right part, while the rest of the gonad consists of ovarian tissue including a follicular cyst. B HE-stained section showing the presence of a primordial follicle in the surrounding ovarian stroma. Positive DDX4 (VASA) staining of the primordial follicle (Inset). C Representative HE staining of the testicular tissue showing the presence of seminiferous tubules with spermatogenesis (right side) next to the seminiferous tubules with Sertoli-cell-only (left side). D DDX4 (VASA) positive spermatogonial cells present in a subset of the seminiferous tubules (right side, brown). E Positive staining of spermatogonia with TSPY (red). F Representative part of the testicular tissue showing absence of OCT3/4 (POU5F1)-positive staining. Positive control (brown, inset). G The granulosa cells in the ovarian stroma and surrounding the primordial follicle show expression of FOXL2 (brown). H Positive SOX9 staining (brown) of the Sertoli cells present in the seminiferous tubules. Note the presence of spermatogenesis in the tubules on the right side next to Sertoli-cell-only tubules (left side). I, J Representative FISH with Y centromere (red) and X centromere (green) probes. Note the presence of $Y$ centromeres in cells surrounding the primordial follicle next to cells showing $2 \mathrm{X}$ centromere signals; within the seminiferous tubules most cells show an $\mathrm{XY}$ constitution. A $\times 5, \mathbf{B}-\mathbf{H} \times 400$.

146

Sex Dev 2018;12:145-154 DOI: $10.1159 / 000479946$ van Bever et al. 
netic explanation of OT-DSD is identified in about $30 \%$ of the patients [Ledig et al., 2012]. For many decades it has been realized that a 46,XX/46,XY chromosomal constitution can be an isolated sex chromosome abnormality related to OT-DSD, in which case the autosomes show a similar molecular constitution in both cell lines [De Marchi et al., 1976; Niu et al., 2002; Alonso et al., 2007]. Alternatively, it can be caused by chimerism, characterized by a different molecular constitution of the autosomes present in the cell lines because of the origin from (at least) 2 different gametes [Zuffardi et al., 1987; Xia et al., 2014].

Here, we report on a male patient with tetragametic chimeric OT-DSD, diagnosed by various approaches, including blood typing as well as genome-wide and targeted SNP analyses. His condition can only be explained by the mixtures of 2 independently fertilized oocytes, one with an $\mathrm{X}$ - and one with a Y-containing sperm, explaining the 2 diploid cell line constitution combined with the presence of up to 4 individual alleles. The patient described underwent a complete unilateral gonadectomy because of suspected cancer and subsequent partial contralateral gonadectomy because of remaining ovarian activity. The testicular part was left in situ to preserve testicular tissue for hormonal activity as well as putative future fertility. Hormonal treatment was applied to subsequently suppress the remaining ovarian activity, resulting in pain relief. The relevance of the genetic and pathological gonadal diagnosis will be discussed in the context of optimal clinical management in this single patient.

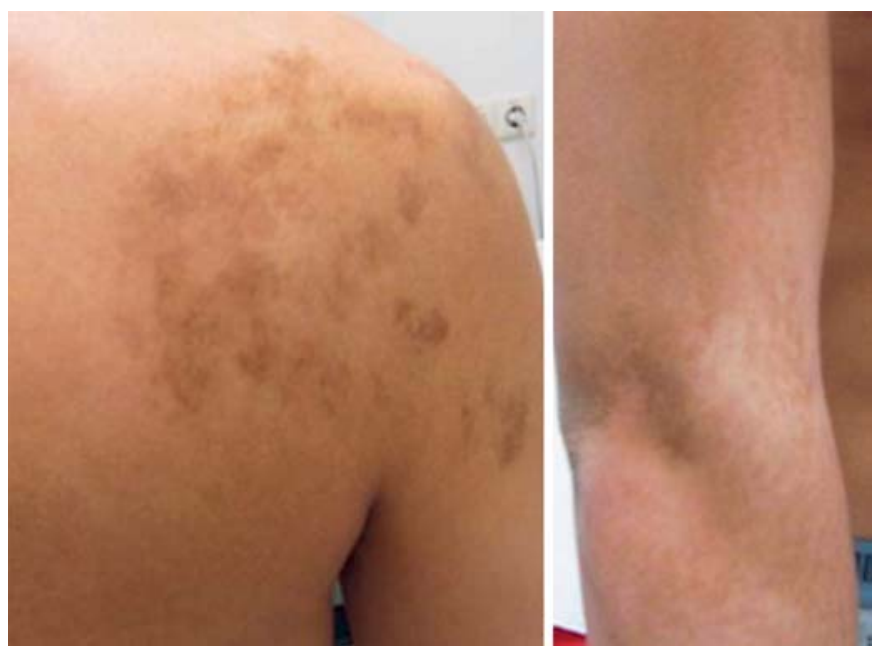

Fig. 2. Representative images of the pigmented areas on the skin known as Blaschko's lines.

Male Tetragametic Chimerism and

Scrotal Ovotesticular DSD

\section{Case Report}

A 19 year-old male, born from a Caucasian father and an Asian mother, presented to a local hospital with a painless right scrotal mass. Unilateral gonadectomy was performed because of suspicion of testicular cancer. Histological investigation at a peripheral pathology institute resulted in the diagnosis of a typical ovotestis (Fig. 1A-C). The patient was referred to the Erasmus MC DSD expert team for further treatment. The gonadal histology was confirmed by the referral pathologist, and additional confirmative immunohistochemical stainings, including DDX4, TSPY and OCT3/4 (POU5F1), SOX9 and FOXL2, were performed (Fig. 1). His medical history was unremarkable, except for an asymmetrical gynecomastia since the onset of puberty at the age of 14 years. He had no history of cryptorchidism. Physical examination showed a slender male, normal virilized phenotype with persisting asymmetric gynecomastia, Tanner stage B4 on the right side and B2 on the left side, normal male external genitalia, a left scrotal gonad with a volume of $12-15 \mathrm{~mL}$ with a normal texture on ultrasound, and an empty right hemi-scrotum after the unilateral gonadectomy. Several striking irregular pigmentations on the right shoulder and upper arm with a distribution reminiscent of Blaschko's lines were identified (Fig. 2).

\section{Endocrinological and Imaging-Based Evaluation}

Laboratory investigations (Table 1) showed elevated gonadotropins and a testosterone level at the high end of the reference range ( $29 \mathrm{nmol} / \mathrm{L}$, reference range $10-30 \mathrm{nmol} / \mathrm{L}$ ). Estradiol and progesterone were within the male reference range, whereas prolactin was elevated $(1.64 \mathrm{U} / \mathrm{L}$, male reference range $0-0.36 \mathrm{U} / \mathrm{L})$. Six weeks after the initial presentation, the patient developed a cystic mass in the upper part of the remaining gonad. MRI of the pelvic region identified an $18 \mathrm{~mm}$ cyst in the gonad close to the epididymis. In addition, a small prostate, a small utriculus, a normal aspect of the left seminal vesicle, an underdeveloped right seminal vesicle, and possible absence of the vasa deferentia were identified. In addition, an uretero pelvic junction stenosis of the right kidney was found, but no signs of malignancy. In the following year, gonadotropin levels fluctuated. Maximum LH was $21.8 \mathrm{U} / \mathrm{L}$ which coincided with the lowest FSH level (12 U/L) and highest estradiol level of $477 \mathrm{pmol} / \mathrm{L}$. At this time, gynecomastia progressed to Tanner stage B3 on the left side. The cystic upper part of the remaining gonad, which measured $1.2 \times 1 \times 0.5 \mathrm{~cm}$ in size, was removed (histological findings are reported below). The aim of this partial surgical intervention was the removal of the ovarian tissue and resulted in regression of breast tissue on the left side. However, 6 months later, the patient had complaints again of intermittent pain/discomfort caused by a palpable gonadal cyst, likely an ovarian follicle. Laboratory investigations showed that estradiol and prolactin levels remained elevated. The patient did not decide for gonadectomy because of the resulting loss of hormonal function and fertility. In addition, he declined the opportunity to investigate his fertility by semen analysis and possibly cryopreservation or testicular sperm extraction (TESE) at that point in life. Therefore, treatment with testosterone was initiated, despite normal testosterone levels, to suppress LH and thereby suppress ovarian activity. During treatment with $50 \mathrm{mg}$ /day transdermal testosterone gel, he no longer experienced gonadal pain, despite LH and estradiol initially remaining elevated, although levels seemed to decline after several months. 
Table 1. Summary of the endocrine investigations

\begin{tabular}{|c|c|c|c|c|c|c|c|c|c|}
\hline & \multicolumn{9}{|c|}{ Time points, weeks } \\
\hline & 0 & 5 & 32 & $45^{\mathrm{a}}$ & 62 & 67 & $73^{b}$ & 100 & 126 \\
\hline Estradiol, pmol/L & 71 & 161 & $477^{\mathrm{H}}$ & $200^{\mathrm{H}}$ & 108 & $192^{\mathrm{H}}$ & $175^{\mathrm{H}}$ & $151^{\mathrm{H}}$ & 88 \\
\hline $\mathrm{TSH}, \mathrm{mU} / \mathrm{L}$ & & & 1.60 & & & & & & \\
\hline Free T4, pmol/L & & & 13.7 & & & 15.5 & & & \\
\hline $\mathrm{LH}, \mathrm{U} / \mathrm{L}$ & $11.9^{\mathrm{H}}$ & $20.7^{\mathrm{H}}$ & $21.7^{\mathrm{H}}$ & 6.7 & $13.4^{\mathrm{H}}$ & $18.2^{\mathrm{H}}$ & $16.1^{\mathrm{H}}$ & $21.8^{\mathrm{H}}$ & $10.0^{\mathrm{H}}$ \\
\hline FSH, U/L & $32.7^{\mathrm{H}}$ & $14.7^{\mathrm{H}}$ & $12.0^{\mathrm{H}}$ & $18.4^{\mathrm{H}}$ & $31.5^{\mathrm{H}}$ & $30.0^{\mathrm{H}}$ & $22.2^{\mathrm{H}}$ & $29.5^{\mathrm{H}}$ & $26.6^{\mathrm{H}}$ \\
\hline $\mathrm{AMH}, \mu \mathrm{g} / \mathrm{L}$ & 5.3 & 5.8 & & 5.6 & 4.9 & 5.5 & & & \\
\hline Inhibin $\mathrm{B}, \mathrm{ng} / \mathrm{L}$ & $32^{\mathrm{L}}$ & & $25^{\mathrm{L}}$ & $18^{\mathrm{L}}$ & $<10^{\mathrm{L}}$ & $22^{\mathrm{L}}$ & & & \\
\hline Prolactin, U/L & $1.64^{\mathrm{H}}$ & $1.7^{\mathrm{H}}$ & $1.4^{\mathrm{H}}$ & $4.1^{\mathrm{H}}$ & $1.06^{\mathrm{H}}$ & $1.45^{\mathrm{H}}$ & $1.17^{\mathrm{H}}$ & $1.13^{\mathrm{H}}$ & $1.0^{\mathrm{H}}$ \\
\hline Progesterone, $\mathrm{nmol} / \mathrm{L}$ & 1.4 & 1.5 & & 1.5 & 1.3 & 1.2 & 1.0 & & \\
\hline Testosterone, $\mathrm{nmol} / \mathrm{L}$ & 29.14 & 19.7 & 18.9 & 18.4 & 23.05 & 24.73 & 13.75 & 29.32 & 26.95 \\
\hline $\mathrm{SHBG}, \mathrm{nmol} / \mathrm{L}$ & & & $73.9^{\mathrm{H}}$ & & & 52.7 & 64.6 & & \\
\hline
\end{tabular}

The time points of the analyses are indicated in weeks after the initial visit to the DSD team (i.e., first measurements were done after unilateral gonadectomy). ${ }^{\mathrm{H}}$, above reference range; ${ }^{\mathrm{L}}$, below reference range; ${ }^{\mathrm{a}}$ Partial gonadectomy of the remaining gonad. ${ }^{\mathrm{b}}$ Start of testosterone treatment.

\section{Materials and Methods}

Blood Typing by Monoclonal Antibodies as well as by DNA Analysis

The ABO blood group typing was done serologically with manually standard gel cards (DiaMed AG, Cressier, Switzerland). Furthermore, an ID-card was used for complete profiling of the Rhphenotype, including K-typing. In addition, ABO genotyping was performed by sequencing. Furthermore, blood group antigen typing was performed by real-time quantitative PCR-based allelic discrimination assays for RHCE, RHD, FY, JK, and K by the department of immunohematology, Sanquin Diagnostic Services, Amsterdam, The Netherlands, using standardized procedures.

\section{Immunohistological Analyses of Gonadal Tissue}

Immunohistochemical staining was performed on formalinfixed paraffin-embedded gonadal samples of $4 \mu \mathrm{m}$ thickness. Briefly, after deparaffinization and 5 min incubation in $3 \% \mathrm{H}_{2} \mathrm{O}_{2}$ for inactivating endogenous peroxidase activity, antigen retrieval was carried out by heating to $120^{\circ} \mathrm{C}$ under pressure up to 0.9 bar in 0.01 M TRIS/0.001 M EGTA (pH 9.0) buffer. After blocking endogenous biotin using the Avidin/Biotin Blocking Kit (SP-2001; Vector Laboratories), sections were incubated either overnight at $4{ }^{\circ} \mathrm{C}$ (TSPY) or for $2 \mathrm{~h}$ at room temperature (OCT3/4, DDX4, SOX9, and FOXL2) and detected using the appropriate biotinylated secondary antibodies and visualized using the avidin-biotin detection and substrate kits (Vector Laboratories). The antibodies directed against OCT3/4, TSPY, DDX4 (VASA), SOX9, and FOXL2 have been described before [Zeeman et al., 2002; Honecker et al., 2004; Hersmus et al., 2008; Lau et al., 2009].

\section{Genetic Investigations}

Standard karyotyping on cultured lymphocytes was performed, as well as FISH using centromeric probes for the X (CEPX) and the Y chromosome (CEPY) on a buccal smear and on both the ovarian and testicular tissue from the removed gonadectomy specimen.
FISH on tissue slides was performed as follows: slides of $5 \mu \mathrm{m}$ thickness were deparaffinized and heated under pressure of up to 0.9 bar in appropriate buffer (0.001 M TRIS/EGTA, pH 9). Slides were digested using $0.01 \%$ pepsin (Sigma Aldrich) in $0.02 \mathrm{M} \mathrm{HCl}$ at $37^{\circ} \mathrm{C}$, with an optimal digestion time of $1 \mathrm{~min}$. Slides were rinsed and dehydrated, and the probes dissolved in hybridization mixture were applied. Probes for centromere X (pBamX5) and centromere Y (DYZ3) were used, labeled with digoxigenin-11-dUTP and biotin-16-dUTP (Roche Diagnostics) using a nick-translation kit (Gibco BRL). After denaturizing $\left(80^{\circ} \mathrm{C}\right.$ for $\left.10 \mathrm{~min}\right)$, hybridization overnight $\left(37^{\circ} \mathrm{C}\right)$, and washing steps, probes were visualized using Cy3-conjugated avidin (1:100, Jackson ImmunoResearch) and Sheep-anti-dig FITC (1:50, Roche Diagnostics) and analyzed using a fluorescent microscope (Leica Microsystems).

DNA profiling was performed using various approaches. The targeted approach, consisting of a set of 15 highly polymorphic markers, distributed over 13 autosomes, and 1 sex chromosomal marker that differentiates between $\mathrm{X}$ and $\mathrm{Y}$ chromosomes, was performed to establish the number of different parental alleles on isolated DNA of peripheral blood lymphocytes (AmpFISTR ${ }^{\circledR}$ Identifiler ${ }^{\circledR}$ PCR Amplification Kit, ThermoFisher Scientific). A multiplex PCR assay for short tandem repeat (STR) loci was applied including the following markers: TPOX (2p25.1pter), D2S1338 (2q35), D3S1358 (3p21.31), FGA (4q31.3), D5S818 (5q23.2), CSF1P0 (5q33.3), D7S820 (7q21.11), D8S1179 (8q24.13), TH01(11p15.5), vWA (12p12-pter), D13S317 (13q31.1), D16S539 (16q24.1), D18S51 (18q21.33), D19S433(19q12), D21S11(21q21.1), and Amelogenin (Xp22.2p22.3 and Yp11.2). Samples were run on an ABI 3100 (ThermoFisher Scientific) and were analyzed using Genemarker software (SoftGenetics). The genome-wide analysis was performed using the Infinium CytoSNP-HC850v1.1 BeadChip (HC850) array according to the Infinium SNP protocol with an input of $200 \mathrm{ng}$ DNA. Analysis was performed with GenomeStudio v2011.1 (Illumina) and NexusCopyNumber v8 (BioDiscovery). BioDiscovery's SNP-Rank Segmentation Algorithm, an extension of the Rank Segmentation algorithm, was used to make 
copy number as well as loss of heterozygosity (LOH) calls. SNPRank Segmentation takes into account both the log-R as well as the $\mathrm{B}$-allele frequency value at each probe location to create a segment. The significance threshold for segmentation was set at $5 \times 10^{-8}$, also requiring a minimum of 6 probes per segment and a maximum probe spacing of $1,000 \mathrm{~kb}$ between adjacent probes before breaking a segment. The log ratio thresholds for single copy gain and single copy loss were set at 0.18 and 0.16 , respectively. The homozygous frequency threshold was set to 0.85 , the homozygous value threshold was set to 0.8 , the heterozygous imbalance threshold was set to 0.4 , and the minimum $\mathrm{LOH}$ length was set at 1,000 $\mathrm{kb}$. Moreover, a targeted amplicon primer set was applied, containing 2 constitutions, including 197 (experiment 1) and 330 regions (experiment 2) across the genome of which many were informative based on frequent heterozygous markers designed with Ion AmpliSeq ${ }^{\mathrm{TM}}$ Designer. Library preparations were performed with the AmpliSeq Library Kit 2.0-384 LV. Template preparation and sequencing were done with the Ion PGM Hi-Q Chef Kit on an Ion $318 \mathrm{v} 2$ chip. Sequence information was analyzed with Variant Caller v4.4.2.1 (ThermoFisher Scientific), and variants were annotated in a local Galaxy pipeline using ANNOVAR (human genome build 37). All heterozygous positions (5-95\%) were visually inspected [proper alignments, no repeats, no homopolymeric stretches, and sufficient reads $(>50)]$. The average coverage was approximately 800 reads for both experiments. Lesser allele frequencies (LAF) were determined for both experiments and data were averaged.

\section{Results}

\section{Blood Typing}

Blood grouping showed a double population of $\mathrm{O}$ and $\mathrm{AB}$ erythrocytes (Fig. 3) without a history of blood transfusion. The presence of both $\mathrm{A}$ and $\mathrm{B}$ antigens was concordant with the absence of anti-A and anti-B antibodies. DNA genotyping supported the serological findings. The main blood group was $\mathrm{O}, \mathrm{K}$ negative and $\mathrm{K}$ (a) positive, while $15 \%$ of the erythrocytes expressed both blood groups $\mathrm{A}$ and $\mathrm{B}$ and were positive for $\mathrm{K}$ antigen and negative for $\mathrm{K}(\mathrm{a})$ antigen. This indicted the presence of 4 alleles, in line with a tetragametic chimerism of which the O cell line represented $85 \%$ and the $\mathrm{AB}$ cell line $15 \%$.

\section{Gonadal Histology}

As indicated, histological examination of the right gonad obtained after complete gonadectomy showed an ovotestis, characterized by a follicular cyst present in an ovarian stromal context as well as testicular tissue composed of seminiferous tubules with Sertoli-cell-only and tubules with spermatogenesis (representative images of hematoxylin and eosin stained slides and immunohistochemical evaluation are shown in Figure 1A-H). Immunohistochemical staining for DDX4 showed the presence

Male Tetragametic Chimerism and Scrotal Ovotesticular DSD

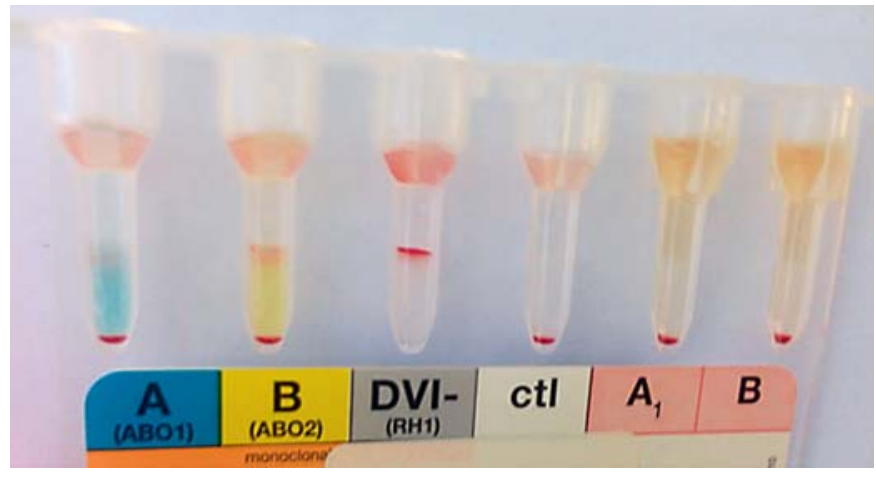

Fig. 3. ID-card (DiaMed, Cressier, Switzerland) for blood groups $\mathrm{ABO}$ and rhesus $\mathrm{D}$ with mononclonal antibodies, showing a mixed field agglutination pattern indicating the presence of $O$ group cells and the simultaneous presence of erythrocyte populations with a weak expression of A and B antigens. Anti-A1 and anti-B antibodies were absent. No mixed field was present for rhesus D.

of germ cells at different stages of maturation, supported by the positive staining for TSPY. The absence of staining for OCT3/4, specific for embryonic stem and germ cells, is in line with the morphological interpretation of the absence of the pre-malignant lesion of germ cell cancers, known as germ cell neoplasia in situ (GCNIS) [Berney et al., 2016]. In addition, SOX9 stained the Sertoli cells in the testicular area of the gonad and FOXL2 the granulosa cells in the ovarian part. Similar observations were made on the partial surgical specimen of the contralateral gonad. In brief, it contained gonadal tissue including seminiferous tubules without germ cells (i.e., Sertoli-cell-only), as well as multiple, although limited, numbers of primordial follicles. In addition, a regressive structure, likely related to a regressive follicle, was identified similar to the gonadectomy specimen (Fig. 1).

\section{Genetic Investigations}

The cultured leucocyte analysis demonstrated a 46,XX karyotype in 23 cells (77\%) and a 46,XY karyotype in 7 cells $(23 \%)$, indicating a mosaic chromosomal constitution, with a XX predominance. FISH with centromeric $\mathrm{X}$ and $Y$ probes on a buccal smear confirmed this observation, with the following distribution: CEPX $\times 2$ in $16 / 50$ (32\%) and CEPX,CEPY $\times 1$ in 34/50 (68\%) cells, showing an XY predominance, in contrast to the investigated leukocytes. FISH with the CEPX and CEPY probes on gonadal tissue showed predominantly XX cells in the ovarian tissue and XY in the testicular part; however, both tissues contained both sex chromosomal patterns (Fig. 1I, $\mathrm{J})$. The results are summarized in Table 2 . Based on the 
Fig. 4. Representative example of the pattern of the marker D18S51 in peripheral blood DNA of the father (upper panel, 2 alleles), the mother (middle panel, 2 alleles), and the patient (lower panel). Note the presence of 4 alleles in the latter, of which the red-circled alleles are the minor in distribution, representing between 10$20 \%$ of the total.

Table 2. Summary of the molecular analyses performed

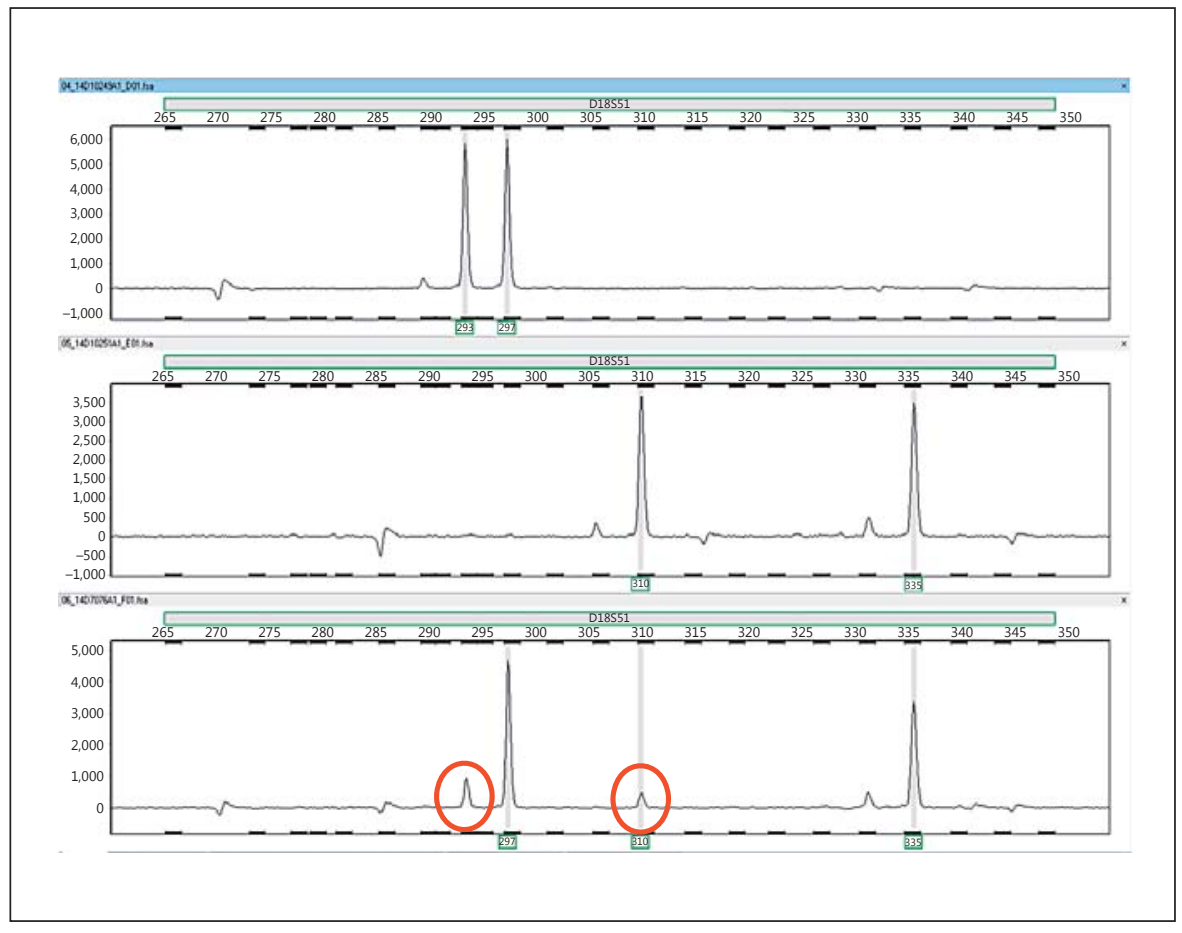

\begin{tabular}{ll}
\hline Test & Result \\
\hline Karyotyping on leucocytes & $46, \mathrm{XX}[23] / 46, \mathrm{XY}[7]$ \\
\hline FISH on buccal smear & $\mathrm{CEPX} \times 2[16 / 50] / \mathrm{CEPX}, \mathrm{CEPY} \times 1[34 / 50]$ \\
\hline $\begin{array}{l}\text { FISH on ovarian and testicular part of } \\
\text { gonad, respectively }\end{array}$ & $\mathrm{CEPX} \times 2 / \mathrm{CEPX}, \mathrm{CEPY} \times 1$ \\
\hline Marker D18S51 & 4 alleles, 2 paternal and 2 maternal \\
\hline Markers TPOX, D2S1338, D3S1358 & extra paternal allele \\
\hline Markers D5S818, D7S820 & extra maternal allele \\
\hline Marker amelogenin & higher signal \\
\hline ABO blood grouping and phenotyping & O K- Jk(a)+/ AB K+ Jk(a)- \\
\hline SNP array and targeted sequencing & aberrant distribution of B allele \\
\hline
\end{tabular}

recurrent XX/XY karyotype, further investigations were initiated using both a targeted as well as genome-wide SNP-based approach. For the targeted analyses, DNA from the patient was compared with DNA of both parents using a set of highly polymorphic markers. Six markers were found to be informative (TPOX, D2S1338, D3S1358, D5S818, D7S820, D18S51). The patterns of the markers TPOX, D2S1338, and D3S1358 were informative for the presence of an additional paternal allele, whereas the patterns of the markers D5S818 and D7S820 demonstrated the presence of an additional maternal allele. The sex chromosomal marker Amelogenin showed a more intense $\mathrm{X}$ chromosomal specific allele in the patient, compared to the $\mathrm{X}$ chromosomal allele in his father (see Table 2 for summary). Marker D18S51 was indicative for the presence of 4 different alleles, 2 of paternal and 2 of maternal origin (Fig. 4). In the DNA of the patient 2 major alleles are observed, 1 paternal and 1 maternal. The left minor paternal allele in the patient overlaps with a specific peak preceding the major paternal allele, but its in- 


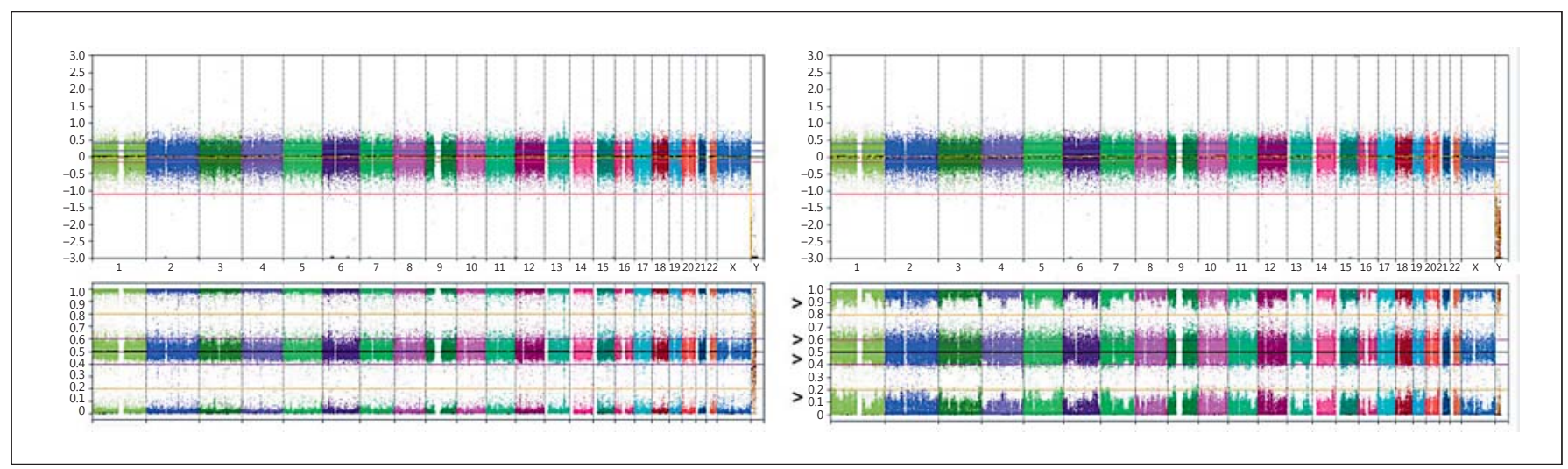

Fig. 5. HC850 SNP array analysis of a normal control 46,XX (left panel) and the 46,XX/46,XY chimeric male patient (right panel) showing the multiple ( 3 or 4 ) alleles at loci throughout the genome. Arrow heads indicate the broad bands of the B-allele frequencies. The upper panels indicate the overall relative copy numbers.

Fig. 6. Representation of the combined molecular data of the tetragametic patient. Array SNP B-allele frequencies (AF) are plotted in grey dots (similar to the information indicated in Figure 5, right panel). The filled arrow heads indicate the major homozygous SNPs. The open arrow heads indicate minor heterozygous frequency groups. The asterisks mark regions lacking the minor frequency groups on multiple chromosomes. The LAF data (right axis) at heterozygous positions are obtained from targeted sequencing of 3 normal peripheral blood DNA samples (white circles) and the patient DNA blood derived sample (red circles). The status of the various polymorphic markers tested is shown with diamonds at their genomic position. $+\mathrm{M}$, additional maternal allele, $+\mathrm{P}$, additional paternal allele, $+\mathrm{PM}$, additional paternal and maternal alleles; NI, noninformative.

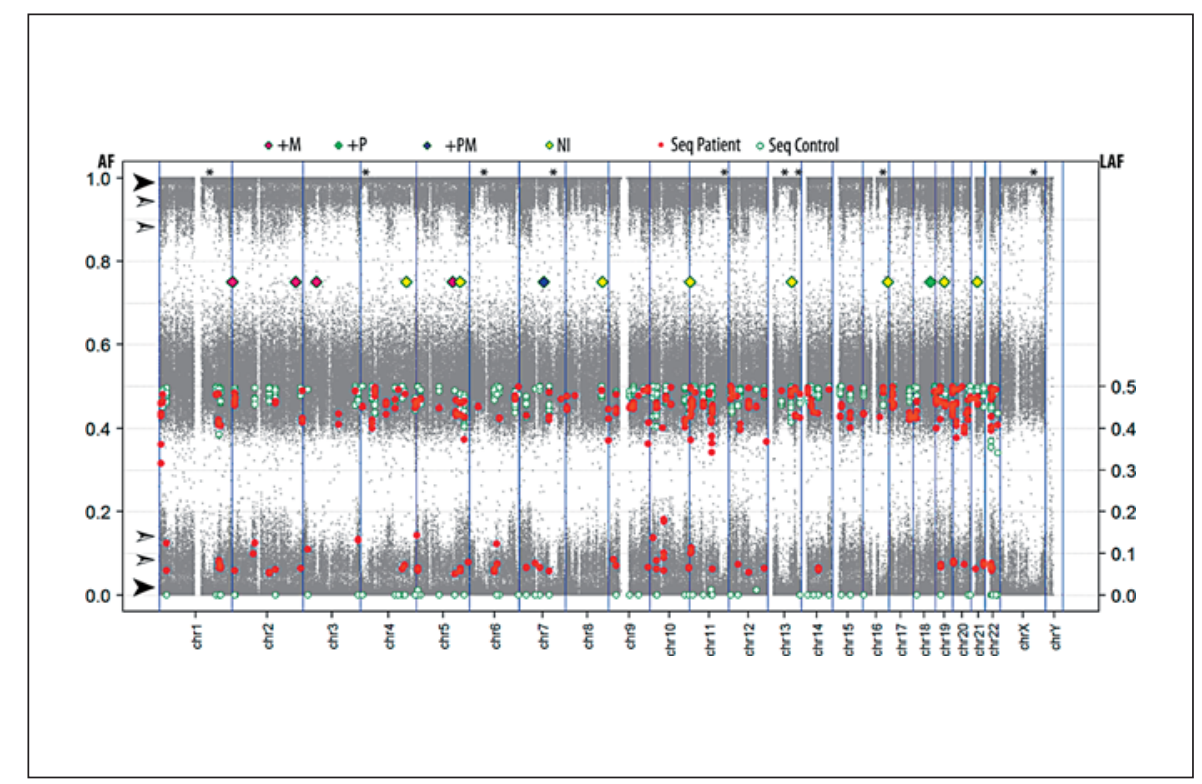

tensity exceeds normal values. The minor alleles represent between $10-20 \%$ of the total. These findings again support a tetragametic chimeric constitution. In parallel, a genome-wide SNP array was performed, showing an aberrant profile compared to a normal control (Fig. 5). The pattern indicated that besides a major population, composed of 2 alleles (either homozygous or heterozygous), a minor population is identified. In addition, the results of the targeted Ion Torrent sequencing approach were overall in line with the array observations. It showed that a relative large group of low frequency alleles (81 SNPs on 48 amplicons, average LAF of 6.6\%) is present in

Male Tetragametic Chimerism and Scrotal Ovotesticular DSD blood-derived DNA, as well as a group of 15 genomic positions (9 amplicons on 7 chromosomes) with an average LAF of $12.7 \%$ (Fig. 6). For several chromosomes, regions with either frequency are present. Of interest is the fact that none of these low frequency domains map to the various regions showing an additional pattern of complexity in the SNP array data with (partial) loss of one of the heterozygous SNPs of the minor population (Fig. 6, regions marked with an asterisk). Overall, these data are again in line with the presence of 4 alleles, with a major as well as a minor cell population, the latter being estimated to be around $12 \%$ of the total. 


\section{Discussion}

The male patient described here had no sign of atypical genitalia and had scrotal gonads, but he was diagnosed with bilateral OT-DSD. A profound genetic and blood typing investigation revealed a defined genetic constitution, tetragametic chimerism, likely explaining the existence of bilateral ovotestes. The first gonad was surgically removed because of suspicion of testicular cancer due to painless enlargement. The pathological diagnosis of the ovotestis led to the referral to the DSD expert team. Of special clinical interest is that the presence of gynecomastia, persisting after puberty, was the only physical indication of an underlying DSD, as reported before [van Niekerk, 1976]. Another study also described gynecomastia in an XX/XY individual without atypical genitalia [Alonso et al., 2007]. The subsequent observed formation of gonadal cysts leading to enlargement of the remaining scrotal gonad after complete unilateral gonadectomy has also been previously reported in a 13 years-old patient, also showing (recurrent) gynecomastia [Cook and Gashti, 1979]. Moreover, the presence of lighter and darker skin pigmented patches in a male with ambiguous genitalia following the Blaschko's lines, a XX/XY chromosomal pattern, and OT-DSD has been reported before as well [Ramsay et al., 2009]. These observations match the specific combination of characteristics identified in the patient described here, i.e., gynecomastia, (recurrent) gonadal cysts, and the Blaschko's lines. Besides these similarities to reported cases, there are a number of additional remarkable as well as clinically and scientifically significant observations which will be discussed hereafter.

The results of the endocrine investigations at the time of the initial evaluation by the DSD team (i.e., after the unilateral gonadectomy) were unexpected for an assumed normal male. Both FSH and LH levels were elevated. The high-normal testosterone level suggested that this could not be completely attributed to gonadal failure. The peak of $\mathrm{LH}$, associated with an LH/FSH ratio of 1.8, was suggestive of a female-type LH peak related to positive feedback to the pituitary from elevated estradiol concentrations produced by an ovarian follicle. The generated LH peak may theoretically result in ovulation, which could explain the recurrent gonadal pain the patient experienced. Indeed, ovulation-suggestive features have been reported in scrotal ovotestes in a male adolescent [Kropp et al., 1995]. In fact, it has been reported that ovulation occurs in half of all ovotestes [van Niekerk, 1976]. The elevated prolactin levels are likely secondary to hyperestrogenism, as chronically elevated levels of estradiol are known to stimulate prolactin secretion [Grattan and Kokay, 2008]. Elevated prolactin levels have previously been observed in males with high estradiol levels, for example due to a feminizing adrenal tumor [Subbiah et al., 2011]. Hyperprolactinemia has also been reported in up to $4 \%$ of transgender women (femaleidentifying individuals assigned male at birth) treated with estrogens [Wierckx et al., 2014].

Clinical treatment of the complaints related to recurrent symptomatic ovotestes, as present in the patient described, may consist of partial gonadectomy. This requires complete removal of ovarian tissue whilst saving as much testicular tissue as possible. This may allow retention of hormonal activity and might be sufficient for future fertility as well. The risk for germ cell malignancy in OT-DSD is overall low, as reported before, for which the identification of the precursor lesion, either GCNIS or gonadoblastoma, both characterized by a positive staining for OCT3/4 (POU5F1), is informative [van der Zwan et al., 2015; Hersmus et al., 2017; Wolffenbuttel et al., 2017]. OCT3/4 staining was negative in the testicular areas of our patient, in line with a low risk. As demonstrated here and previously reported [Kropp et al., 1995], one may not succeed in removing all ovarian tissue during partial gonadectomy, because of the approach to retain testicular tissue as much as possible. The results described illustrate that the ovarian activity and associated complaints of painful gonadal swelling can be successfully suppressed by hormonal treatment, i.e., testosterone application, in spite of a normal endogenous testosterone level. This is a relevant alternative intervention compared to surgery to be discussed with the patients involved. Theoretically, an aromatase inhibitor can effectively reduce estradiol levels as well but may induce polycystic ovarian tissue that through increase in size may compromise adjacent testicular tissue and therefore was not considered a suitable option. In conclusion, testosterone supplementation was successful in the patient to resolve his gonadal pain, although LH and estradiol levels were not fully suppressed. Addition of a GnRH analogue would more effectively suppress gonadotropins but was not applied because the patient experienced no complaints anymore.

Multiple studies have been reported related to a $46, \mathrm{XX} / 46$, XY karyotype in humans, although not all investigated whether it concerned an isolated sex chromosomal disorder or chimerism. Some older reports refer to true hermaphroditism, although without histological characterization of the $\operatorname{gonad}(\mathrm{s})$, so that the diagnosis
152

Sex Dev 2018;12:145-154 DOI: $10.1159 / 000479946$ van Bever et al. 
ovotestes cannot be made with certainty. Theoretically, karyotyping in multiple tissues, blood typing, and SNPs can all be used to investigate the molecular constitution of XX/XY patients. The karyotyping in the patient described was performed on cultured peripheral blood lymphocytes as well as buccal smears. A difference in distribution was found, with a predominance of the $46, \mathrm{XX}$ cell line in the first and of the $46, \mathrm{XY}$ cell line in the latter. This can be related to culturing of the lymphocytes or due to an actual difference in distribution in the different tissues. Of interest is that both the blood typing results and the SNP profiling support the predominance of the 46,XX clone as found in the cultured lymphocytes. This also matches the findings of the $\mathrm{X}$ and $\mathrm{Y}$ distributions found in the gonadal tissue, although a predominance of the 46,XX cell line was observed in the ovarian and the $46, \mathrm{XY}$ cell line in the testicular area. The approaches applied are informative to detect chimeras in case of similar sex chromosomal constitution of the various cell lines. This is important as detailed follow-up protocols exist for specific variants of DSD patients, paying attention to their precise health risks. Moreover, identification can have significant advantages, for example related to the type of donor blood that is suitable in cases of an emergency because of the putative presence of different blood types. All of the results of the analyses performed in the patient described support the presence of chimerism and not sex chromosome mosaicism. In addition, multiple tests demonstrated the presence of 4 alleles, although in a different ratio. Various mechanisms are proposed to result in a tetragametic chimerism, explaining the number of different alleles [Strain et al., 1995, Giltay et al., 1998]. These include fertilization of 2 different oocytes by 2 sperm, followed by fusion of the 2 early stage embryo's, resulting in a single tetragametic chimeric individual. Alternatively, it can result from the fertilization of both the oocyte and the polar body by 2 different sperm. Based on the specific SNP profile, identified both by the targeted as well as genome-wide approach and supported by the blood typing results, the first mechanism is the most likely because of the complexity of the ratio of the different alleles. In fact, it is expected that the second mechanism will result in a balanced ratio of all heterozygous SNPs throughout the genome, because redistribution of the informative SNPs over the chromosomal homologues does not occur post-meiotically. From a clinical genetics point of view, SNP array analysis is widely used and may coincidentally demonstrate more than 2 SNPs per locus within a single individual. Possible contamination of

Male Tetragametic Chimerism and Scrotal Ovotesticular DSD foreign DNA must be excluded in such cases, which is easily performed by retesting a new blood sample and/ or, as demonstrated here, by comparison to parental DNA.

In addition to the diagnostic procedures and clinical management described, psychological counseling is warranted for patients, caretakers, and families. As in the patient described, ovotestes and/or chimerism are often diagnosed later in life as patients have been born with typical male or female external genitals. The presence of ovotestes, chimerism, or mosaicism (of the sex chromosomes) may evoke confusion and discussion about one's gender in patients and parents. Gender mixup is extremely stressful as patients and parents experience a loss of identity and loss of all basic knowledge about themselves/their personality (their child/their child's personality). While informing patients and parents about the diagnostic findings, it is important that gender development is discussed in the same session by a well-informed professional. It should be made clear that the genetic findings or pathological evaluation does not mean that there is any doubt about the patient's gender (the patient's experiences of being a male or female person). It should be explained that sexual and gender determination and differentiation are complex, multifactorial processes determined by genes, steroid action, and upbringing and that the karyotype alone is not allimportant in gender development [Callens et al., 2016; Hines et al., 2016].

\section{Summary and Conclusions}

A tetragametic chimeric phenotypical male patient is described with bilateral scrotal ovotestis with ovulatory activity. Gynecomastia initially was the only clinical sign of DSD followed by a scrotal enlargement suspected to be cancer. The genetic diagnosis was based on blood typing as well as SNP investigations and was supported by hyperpigmentation following the Blaschko's lines. The patient was treated by (partial) gonadal surgery, followed by hormonal treatment, with satisfactory results up to now. However, it remains challenging to treat symptoms, preserve gonadal hormonal function and possibly fertility, and manage the risk of malignant transformation. This case illustrates the power of a dedicated multidisciplinary team in the diagnostic procedures and clinical management of individual patients, striving to fulfill their personal needs by optimally using clinical opportunities.

Sex Dev 2018;12:145-154

DOI: $10.1159 / 000479946$ 


\section{Statement of Ethics}

The patient fully agreed upon publication of the results as reported.

\section{Disclosure Statement}

The authors have no conflicts of interest to declare.

\section{References}

Alonso G, Pasqualini T, Busaniche J, Ruiz E, Chemes H: True hermaphroditism in a phenotypic male without ambiguous genitalia: an unusual presentation at puberty. Horm Res 68:261-264 (2007).

Baetens D, Stoop H, Peelman F, Todeschini AL, Rosseel T, et al: NR5A1 is a novel disease gene for 46,XX testicular and ovotesticular disorders of sex development. Genet Med 19:367376 (2016).

Bashamboo A, Donohoue PA, Vilain E, Rojo S, Calvel P, et al: A recurrent p.Arg92Trp variant in steroidogenic factor-1 (NR5A1) can act as a molecular switch in human sex development. Hum Mol Genet 25:3446-3453 (2016).

Berney DM, Looijenga LH, Idrees M, Oosterhuis JW, Rajpert-De Meyts E, et al: Germ cell neoplasia in situ (GCNIS): evolution of the current nomenclature for testicular pre-invasive germ cell malignancy. Histopathology 69:710 (2016).

Callens N, Van Kuyk M, van Kuppenveld JH, Drop SL, Cohen-Kettenis PT, et al: Recalled and current gender role behavior, gender identity and sexual orientation in adults with disorders/differences of sex development. Horm Behav 86:8-20 (2016).

Conlin LK, Thiel BD, Bonnemann CG, Medne L, Ernst LM, et al: Mechanisms of mosaicism, chimerism and uniparental disomy identified by single nucleotide polymorphism array analysis. Hum Mol Genet 19:1263-1275 (2010).

Cook WA, Gashti E: Asymmetrical gonadal enlargement in adolescent true hermaphrodite with bilateral ovotestes. Urology 13:63-66 (1979).

De Marchi M, Carbonara AO, Carozzi F, Massara F, Belforte L, et al: True hermaphroditism with XX/XY sex chromosome mosaicism: report of a case. Clin Genet 10:265-272 (1976).

Giltay JC, Brunt T, Beemer FA, Wit JM, van Amstel HK, et al: Polymorphic detection of a parthenogenetic maternal and double paternal contribution to a $46, \mathrm{XX} / 46, \mathrm{XY}$ hermaphrodite. Am J Hum Genet 62:937-940 (1998).

Grattan DR, Kokay IC: Prolactin: a pleiotropic neuroendocrine hormone. J Neuroendocrinology 20:752-763 (2008).
Hersmus R, Kalfa N, de Leeuw B, Stoop H, Oosterhuis JW, et al: FOXL2 and SOX9 as parameters of female and male gonadal differentiation in patients with various forms of disorders of sex development (DSD). J Pathol 215:31-38 (2008).

Hersmus R, van Bever Y, Wolffenbuttel KP, Biermann K, Cools M, Looijenga LH: The biology of germ cell tumors in disorders of sex development. Clin Genet 91:292-301 (2017).

Hines M, Pasterski V, Spencer D, Neufeld S, Patalay $\mathrm{P}$, et al: Prenatal androgen exposure alters girls' responses to information indicating gender-appropriate behaviour. Philos Trans R Soc Lond B Biol Sci 371:20150125 (2016).

Honecker F, Stoop H, de Krijger RR, Chris Lau YF, Bokemeyer C, Looijenga LH: Pathobiological implications of the expression of markers of testicular carcinoma in situ by fetal germ cells. J Pathol 203:849-857 (2004).

Igarashi $\mathrm{M}$, Takasawa $\mathrm{K}$, Hakoda $\mathrm{A}$, Kanno J, Takada S, et al: Identical NR5A1 missense mutations in two unrelated 46,XX individuals with testicular tissues. Hum Mutat 38:39-42 (2017).

Kropp BP, Keating MA, Moshang T, Duckett JW: True hermaphroditism and normal male genitalia: an unusual presentation. Urology 46: 736-739 (1995).

Lau YF, Li Y, Kido T: Gonadoblastoma locus and TSPY gene on the human Y chromosome. Birth Defects Res C Embryo Today 87:114122 (2009).

Lee HJ, Yoon SC, Ko JM, Seong MW, Park SS, et al: Monochorionic dizygotic twins with discordant sex and confined blood chimerism. Eur J Pediatr 173:1249-1252 (2014).

Ledig S, Hiort O, Wünsch L, Wieacker P: Partial deletion of DMRT1 causes 46,XY ovotesticular disorder of sexual development. Eur J Endocrinol 167:119-124 (2012).

Niu DM, Pan CC, Lin CY, Hwang B, Chung MY: Mosaic or chimera? Revisiting an old hypothesis about the cause of the $46, \mathrm{XX} / 46, \mathrm{XY}$ hermaphrodite. J Pediatr 140:732-735 (2002).
Ramsay M, Pfaffenzeller W, Kotze E, Bhengu L, Essop F, de Ravel T: Chimerism in black Southern African patients with true hermaphroditism 46,XX/47XY,+21 and 46,XX/ 46,XY. Ann NY Acad Sci 1151:68-76 (2009).

Strain L, Warner JP, Johnston T, Bonthron DT: A human parthenogenetic chimaera. Nat Genet 11:164-169 (1995).

Subbiah S, Walia R, Kumar S, Nahar U, Bhansali A: An unusual cause of gynaecomastia. BMJ Case Rep pii:bcr0420114154 (2011).

van der Zwan YG, Biermann K, Wolffenbuttel KP, Cools M, Looijenga LH: Gonadal maldevelopment as risk factor for germ cell cancer: towards a clinical decision model. Eur Urol 67:692-701 (2015).

van Niekerk WA: True hermaphroditism: an analytic review with a report of 3 new cases. Am J Obstet Gynecol 126:890-907 (1976).

Wierckx K, Van CE, Schreiner T, Haraldsen I, Fisher A, et al: Cross-sex hormone therapy in trans persons is safe and effective at shorttime follow-up: results from the European network for the investigation of gender incongruence. J Sex Med 11:1999-2011 (2014).

Wolffenbuttel K, Looijenga L: Response to commentary to 'Gonadal dysgenesis in disorders of sex development (DSD): Diagnosis and surgical management'. J Pediatr Urol 13:116 (2017).

Xia XY, Wang WP, Li TF, Li WW, Wu QY, et al: A parthenogenetic maternal and double paternal contribution to an ovotesticular disorder of sex development. Mol Cytogenet 7:16 (2014).

Zeeman AM, Stoop H, Boter M, Gillis AJ, Castrillon DH, et al: VASA is a specific marker for both normal and malignant human germ cells. Lab Invest 82:159-166 (2002).

Zhu PY, Yan JM, Xue M, Ye D, Yao GH, Luan JF: A case of dispermic chimerism with normal phenotype identified during $\mathrm{ABO}$ blood grouping. Clin Lab 60:1049-1054 (2014).

Zuffardi O, Gargantini L, Lambiase S, Lo Curto F, Maraschio P, Ford CE: Presumptive mosaic origin of an $\mathrm{XX} / \mathrm{XY}$ female with ambiguous genitalia. J Med Genet 24:177-180 (1987). 